\title{
Reviewer Acknowledgements for Global Journal of Health Science, Vol. 12, No. 11
}

Global Journal of Health Science wishes to acknowledge the following individuals for their assistance with peer review of manuscripts for this issue. Their help and contributions in maintaining the quality of the journal are greatly appreciated.

Global Journal of Health Science is recruiting reviewers for the journal. If you are interested in becoming a reviewer, we welcome you to join us. Please contact us for the application form at: gjhs@ccsenet.org.

\section{Reviewers for Volume 12, Number 11}

Abiodun Adeniran, University of Ilorin, Nigeria

Ama Pokuaa Fenny, University of Ghana, Ghana

Angel Alfonso Velarde Lopez, University of Pennsylvania, Guatemala

António Calha, Polytechnic Institute of Portalegre, Portugal

Ayesha Johnson, University of South Florida, United States of America

David John Lindsay, James Cook University, Australia

Emad Adel Shdaifat, Imam Abdulrahman Bin Faisal University, Saudi Arabia

Farahnaz Amini, UCSI University, Malaysia

Francisco Rodenas Rigla, University of Valencia, Spain

Gabriel Gulis, University of Southern Denmark, Denmark

Gabriele Messina, University of Siena, Italy

Georgann Valerie Weissman, Capella University, United States of America

Horng-Jyh Tsai, Kuang Tien General Hospital, Taiwan

Hülya YARDIMCI, Ankara University, Turkey

José Joaquín Mira, Universidad Miguel Hernández, Spain

Kartheek R Balapala, University Tunku Abdul Rahman, Malaysia

Marcel Wullschleger, University of Bern, Switzerland

Meng Zhao, Texas A\&M University at Corpus Christi, United States of America

Myo Nyein Aung, Boromrajonani College of Nursing, Thailand

Pedram Iranmanesh, Dentist, Independent Researcher, Iran

Pi-Ming Yeh, Missouri Western State University, United States of America

Polly Yeung, Massey University, New Zealand

Samir Othman, Hawler Medical University, Iraq

Soon Soo Hoo, Royal North Shore Hospital, Australia

Steven Hoffman, Brigham Young University, United States of America

Thanusin Saleeon, Ministry of Public Health, Thailand 